\title{
Interference Alignment Through Staggered Antenna Switching for MIMO BC With No CSIT
}

\author{
Chenwei Wang, Tiangao Gou, Syed A. Jafar \\ Electrical Engineering and Computer Science \\ University of California, Irvine, Irvine, CA, USA \\ Email: \{chenweiw, tgou, syed\}@uci.edu
}

\begin{abstract}
In this paper, we explore the degrees of freedom (DoF) of the broadcast channel (BC) where the transmitter is equipped with $M$ antennas and there are $K$ receivers, each equipped with $N$ reconfigurable antennas capable of switching among $M$ preset modes. Without any knowledge of the channel coefficient values but only receiver antenna switching modes at the transmitter, we propose an interference alignment scheme for this channel. We show that if $N<M$, then a total of $\frac{M N K}{M+K N-N}$ DoF are achievable, almost surely. The key to this interference alignment scheme is the ability of the receivers to switch between reconfigurable antenna modes to create short term channel fluctuation patterns that are exploited by the transmitter. Compared to the results we showed for MISO BC [6], the supersymbol of MIMO BC may have diverse structures depending $M$ and $N$, and it can be determined from that of MISO BC through an iterative mapping function.
\end{abstract}

\section{INTRODUCTION}

Multiple antenna system has a potential to increase capacity by providing more spatial DoF, whose availability depends on two factors: cooperation within inputs and outputs, and channel knowledge. Although the effect of inability to jointly process the signals at distributed outputs on the DoF is well known with perfect channel state information, the effect of the absence of channel information is less understood. Consider a $K$ user MIMO BC with $M$ antennas at the transmitter and $N$ antennas at each receiver. With channel state information at the transmitter (CSIT), this channel has a total of $\min (M, K N)$ DoF. However, if there is no CSIT, then we only know the DoF of some certain cases. One case is the compound channel [1], [2], [5] where the channel vector is drawn from a finite set which is known at the transmitter but he does not know the specific value. If the channel coefficients follow i.i.d. Rayleigh fading across antennas, users and time slots, then it has been proved that the time-division between the users is optimal, i.e., $\min (M, N)$ [3]. Note that if $M \leq N$, there is no DoF loss since the outerbound of $\operatorname{DoF} \min (M, K N)=M$ can be achieved even without CSIT. If $N<M$, then it indicates that the number of DoF collapses to $N$, since the transmitter's ability to zero-force interference is lost.

Recently, a breakthough appeared in [4] indicates that even without CSIT, we can still do blind interference alignment if the channel coefficients have some special staggered block coherence structures. In [4], it has been shown that for a two user MISO BC with $M=2$, if the coherence blocks of the two users are suitably staggered, then we can use a simple interference alignment to achieve the outerbound. With this idea, in [6], we proposed a systematic blind interference alignment scheme for the vector broadcast channel where each receiver is equipped with a reconfigurable antenna capable of switching among preset modes. In this paper, we propose a systematic way to achieve blind interference alignment for $K$ user MIMO BC without CSIT through staggered antenna switching (SAS) at the receiver. We show that $\frac{M N K}{M+K N-N}$ DoF can be achieved for the $K$ user $M \times N$ MIMO BC. Compared to the result in [6], this result can also be interpreted as increasing the number of DoF achieved by the $K$ user $\frac{M}{N} \times 1$ MISO BC by $N$ times. However, the achievability is not trivial as it looks due to different possible combinations of $M$ and $N$. In this paper, we propose the SAS patterns and a mapping function between the supersymbols of the MIMO BC [6] and that of its MISO BC counterpart. With SAS patterns and the mapping function, we can easily construct the supersymbol of the $K$ user MIMO BC for blind interference alignment.

The rest of this paper is organized as follows. In Section II, the system model is presented. In Section III, we show the structure of the alignment block. Then a mapping function between the supersymbol structures of MISO BC and MIMO $\mathrm{BC}$ is introduced in Section IV. Finally, the conclusions are summarized in Section V.

\section{SYSTEM MODEL}

Consider a complex $K$ user MIMO BC, where the transmitter has $M$ antennas while each receiver is equipped with $N$ reconfigurable antennas (and thus $N$ RF chains) and each can switch among $M$ preset modes. Let $\mathbf{h}_{j}^{[k]}\left(m_{j}^{[k]}\right)$ denote the $1 \times M$ channel vector associated with the $j^{t h}$ antenna of user $k$ at the mode $m_{j}^{[k]}$ where $j=1, \ldots, N$ and $m_{j}^{[k]} \in \mathcal{M}=$ $\{1, \ldots, M\}$. We assume that the channel vectors are generic, by which we mean that they are drawn from a continuous distribution, so that any $M$ of them are linearly independent almost surely. We assume that the coherence times of the channels are long enough so that the channels stay constant across a supersymbol. Then the channel at user $k$ is represented as $\mathbf{H}^{[k]}\left(\mathbf{m}^{[k]}(t)\right)=\left[\mathbf{h}_{1}^{[k] T}\left(m_{1}^{[k]}(t)\right), \ldots, \mathbf{h}_{N}^{[k] T}\left(m_{N}^{[k]}(t)\right)\right]^{T}$ at time $t$ and $\mathbf{m}^{[k]}(t)=\left[m_{1}^{[k]}(t), \ldots, m_{N}^{[k]}(t)\right]^{T} \in \mathcal{M}^{N}$. Thus, the received signal vector at the $k^{t h}$ user is

$$
\mathbf{y}^{[k]}(t)=\mathbf{H}^{[k]}\left(\mathbf{m}^{[k]}(t)\right) \mathbf{x}(t)+\mathbf{z}^{[k]}(t) \quad k=1, \ldots, K
$$

where $\mathbf{x}(t)$ is an $M \times 1$ transmitted signal vector, and the noise vector $\mathbf{z}^{[k]}(t) \sim \mathcal{C N}\left(\mathbf{0}, \mathbf{I}_{N}\right)$ and i.i.d over time. The channel 
input is subject to an average power constraint $\mathbb{E}\left[\|\mathbf{x}\|^{2}\right] \leqslant P$. We assume that the there is no CSIT except the configurable modes $\mathbf{m}^{[k]}(t)$ while the receiver only knows its own channels.

The transmitter sends independent messages $W^{[k]}$ to user $k$ with rates $R^{[k]}$. A rate tuple $\left(R^{[1]}, R^{[2]}, \ldots, R^{[K]}\right)$ is achievable if every receiver is able to decode its message with arbitrary small error probability. The total number of degrees of freedom $d$ is defined as

$$
d=\lim _{P \rightarrow \infty} \frac{R^{[1]}+\cdots+R^{[K]}}{\log P}
$$

Throughout this paper, we use $a \mid b$ to denote the reminder of $a$ divided by $b$. $\operatorname{det}()$ standards for the determinant operator. The definitions and notations in this paper are all consistent with that we use in [6].

\section{Structure of the Alignment Block}

The following theorem states that we can use SAS to create different temporal correlations at different users to achieve more than $N$ DoF that is achieved without SAS.

Theorem 1: For the $K$ user $M \times N$ MIMO BC with staggered antenna switching, a total of $\frac{M N K}{M+K N-N}$ DoF can be achieved when $M>N$.

First note that if $M \mid N=0$, then the generalization is trivial. For this case, the supersymbol structure is the same as the $K$ user $\frac{M}{N} \times 1$ MISO BC [6]. Then we can obtain the beamforming matrix for the $M \times N$ MIMO BC by replacing the $\frac{M}{N} \times \frac{M}{N}$ identity matrix in the beamforming matrix for the $\frac{M}{N} \times 1$ MISO BC with the $M \times M$ identity matrix. Thus in this section, we only need to show the case when $M \mid N \neq 0$.

Similar to the MISO BC, the supersymbol structure for the MIMO case is obtained by creating non-overlapping alignment blocks. Based on the supersymbol, the beamforming matrix can be obtained. Different from the MISO case, the alignment blocks for the MIMO case may have different structures depending on $M$ and $N$. Before showing the alignment block structure for the general case, we first illustrate this point through three specific examples.

\section{Example 1: 2 User $3 \times 2$ MIMO BC}

The alignment block for the $3 \times 2$ MIMO $\mathrm{BC}$ is shown in Figure 1. The alignment block consists of three symbols, over which the channel changes for the desired user while remains fixed at the undesired users. As shown in Figure 1, the beamforming matrix consists of two block columns, each block is a $3 \times 3$ matrix. Note that this is different from that of MISO case where the beamforming matrix corresponding to one alignment block consists of only one block column. The reason for multiple block columns is to make the six data streams received at the desired receiver to be sent along a square matrix - the effective channel, i.e. the product of channel and beamforming matrices. To see this, consider the following effective channel matrix seen at user 1 , denoted as $\tilde{\mathbf{H}}^{[1]}$. For brevity, we replace $\mathbf{m}^{[1]}(t)$ with different number to stands for the SAS mode and thereinafter.

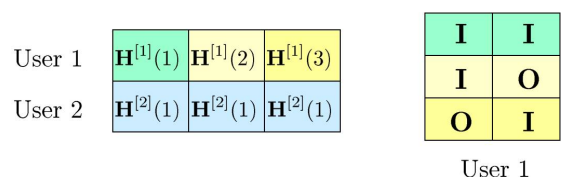

Fig. 1. The alignment block for $3 \times 2$ MIMO BC
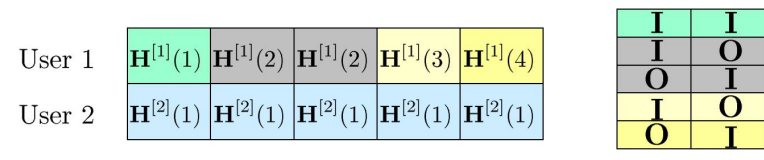

User 1

Fig. 2. The alignment block for $5 \times 2$ MIMO BC

$$
\tilde{\mathbf{H}}^{[1]}=\left[\begin{array}{cc}
\mathbf{H}^{[1]}(1) & \mathbf{H}^{[1]}(1) \\
\mathbf{H}^{[1]}(2) & \mathbf{O} \\
\mathbf{O} & \mathbf{H}^{[1]}(3)
\end{array}\right]_{6 \times 6}
$$

where $\mathbf{O}$ is the $2 \times 3$ zero matrix. Since the channel is a $2 \times 3$ matrix, it is easy to see that the above matrix is a $6 \times 6$ square matrix. Now we need to verify whether the above matrix has full rank, so that user 1 can decode 6 data streams intended for him. In order to achieve this goal, we design the SAS pattern as follows.

$$
\mathbf{H}^{[1]}(1)=\left[\begin{array}{l}
\mathbf{h}_{1}^{[1]} \\
\mathbf{h}_{2}^{[1]}
\end{array}\right], \quad \mathbf{H}^{[1]}(2)=\left[\begin{array}{l}
\mathbf{h}_{3}^{[1]} \\
\mathbf{h}_{1}^{[1]}
\end{array}\right], \quad \mathbf{H}^{[1]}(3)=\left[\begin{array}{l}
\mathbf{h}_{3}^{[1]} \\
\mathbf{h}_{2}^{[1]}
\end{array}\right]
$$

Substitute (4) into $\tilde{\mathbf{H}}^{[1]}$ and apply simple linear transformation. It is easy to see $\operatorname{det}\left(\tilde{\mathbf{H}}^{[1]}\right) \neq 0$, almost surely. Thus, $\tilde{\mathbf{H}}^{[1]}$ is a full rank matrix, almost surely. While at the undesired user, it can also be easily seen that 6 interference streams are aligned into 4 dimensions.

Example 2: 2 User $5 \times 2$ MIMO BC

The alignment block is shown in Figure 2. As we can see, different from the alignment block of the MISO case in which the channel state changes for the desired user, some channels remain fixed for this $5 \times 2$ MIMO BC. Specifically, the alignment block consists of five symbols. It can be divided into three sub-blocks. The first sub-block consists of the first symbol. The second one consists of the second and the third symbols where they remain fixed within this block. The last two symbols constitute the last sub-block in which channel changes for the desired user. With this alignment block, the beamforming matrix is shown in Figure 2. Note that for each block columns, the block rows with the identity matrix see different channels. With such beamforming matrix, the 10 data streams are received along the following $10 \times 10$ matrix.

$$
\tilde{\mathbf{H}}^{[1]}=\left[\begin{array}{cc}
\mathbf{H}^{[1]}(1) & \mathbf{H}^{[1]}(1) \\
\mathbf{H}^{[1]}(2) & \mathbf{O} \\
\mathbf{O} & \mathbf{H}^{[1]}(2) \\
\mathbf{H}^{[1]}(3) & \mathbf{O} \\
\mathbf{O} & \mathbf{H}^{[1]}(4)
\end{array}\right]_{10 \times 10}
$$

The SAS pattern is designed as follow.

$$
\begin{aligned}
\mathbf{H}^{[1]}(1)=\left[\begin{array}{l}
\mathbf{h}_{1}^{[1]} \\
\mathbf{h}_{2}^{[1]}
\end{array}\right], & \mathbf{H}^{[1]}(2)=\left[\begin{array}{c}
\mathbf{h}_{3}^{[1]} \\
\mathbf{h}_{4}^{[1]}
\end{array}\right] \\
\mathbf{H}^{[1]}(3)=\left[\begin{array}{l}
\mathbf{h}_{5}^{[1]} \\
\mathbf{h}_{1}^{[1]}
\end{array}\right], & \mathbf{H}^{[1]}(4)=\left[\begin{array}{l}
\mathbf{h}_{5}^{[1]} \\
\mathbf{h}_{2}^{[1]}
\end{array}\right]
\end{aligned}
$$



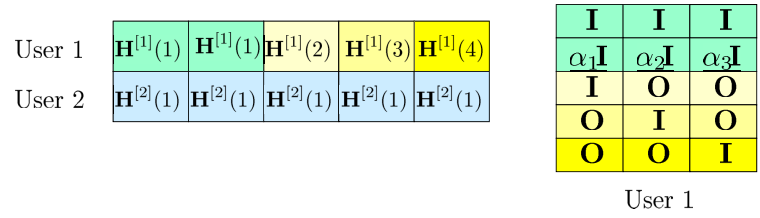

Fig. 3. The alignment block for $5 \times 3$ MIMO BC

With simple linear transformation, it can be seen that $\tilde{\mathbf{H}}^{[1]}$ has full rank, almost surely. Thus, the desired user can separate all desired data streams. It can be also easily seen that at the undesired user, 10 interference streams are aligned into 4 dimensions.

Example 3: 2 User $5 \times 3$ MIMO BC

The alignment block is shown in Figure 3 . It consists of 5 symbols, over which the channel changes at the desired user while remains fixed at the undesired user. The corresponding beamforming matrix is also shown in Figure 3 where $\alpha_{1}, \alpha_{2}$ and $\alpha_{3}$ are scalars independently drawn from a continuous distribution and $\mathbf{I}$ is the $5 \times 5$ identity matrix. Note that there is another difference between the beamforming matrices of the MIMO and MISO cases. For the MISO BC, the beamforming matrix consists of identity matrices. However, for the MIMO $\mathrm{BC}$ some identity matrices are scaled by a random coefficient. The reason is to make sure the desired signals are received linearly independently at the receiver. Consider the following matrix along which the desired signals are received.

$$
\tilde{\mathbf{H}}^{[1]}=\left[\begin{array}{ccc}
\mathbf{H}^{[1]}(1) & \mathbf{H}^{[1]}(1) & \mathbf{H}^{[1]}(1) \\
\alpha_{1} \mathbf{H}^{[1]}(1) & \alpha_{2} \mathbf{H}^{[1]}(1) & \alpha_{3} \mathbf{H}^{[1]}(1) \\
\mathbf{H}^{[1]}(2) & \mathbf{O} & \mathbf{O} \\
\mathbf{O} & \mathbf{H}^{[1]}(3) & \mathbf{O} \\
\mathbf{O} & \mathbf{O} & \mathbf{H}^{[1]}(4)
\end{array}\right]_{15 \times 15}
$$

The SAS pattern is designed as follows.

$$
\begin{aligned}
& \mathbf{H}^{[1]}(1)=\left[\begin{array}{l}
\mathbf{h}_{1}^{[1]} \\
\mathbf{h}_{2}^{[1]} \\
\mathbf{h}_{3}^{[1]}
\end{array}\right], \quad \mathbf{H}^{[1]}(2)=\left[\begin{array}{l}
\mathbf{h}_{4}^{[1]} \\
\mathbf{h}_{5}^{[1]} \\
\mathbf{h}_{1}^{[1]}
\end{array}\right] \\
& \mathbf{H}^{[1]}(3)=\left[\begin{array}{l}
\mathbf{h}_{4}^{[1]} \\
\mathbf{h}_{5}^{[1]} \\
\mathbf{h}_{2}^{[1]}
\end{array}\right], \quad \mathbf{H}^{[1]}(4)=\left[\begin{array}{c}
\mathbf{h}_{4}^{[1]} \\
\mathbf{h}_{5}^{[1]} \\
\mathbf{h}_{3}^{[1]}
\end{array}\right]
\end{aligned}
$$

Again, with linear transformation, it can be easily shown that $\tilde{\mathbf{H}}^{[1]}$ has full rank, almost surely. Thus, the 15 desired data streams are linearly dependent with each other. Also, at the undesired receiver, 15 interference streams are aligned into 9 dimensions.

Now we are ready to present the general case, i.e., the $M \times N$ MIMO BC. The alignment block consists of $M$ symbols as shown in Figure 4. Since the channel remains constant at all undesired user, we omit it in the figure for simplicity. To understand the structure of alignment block at the desired user, we can further divide it into $\left\lfloor\frac{M}{N}\right\rfloor+1$ sub-blocks. As shown in Figure 4, the first sub-block consists of $M \mid N$ symbols. While the channel keeps unchanged across different symbols in the first sub-block, we generate coefficients i.i.d. for each identity block matrices in the beamforming matrix (denoted by $\star$ in the figure) to satisfy the full rank requirement at the desired receiver. Each of the remaining $\left\lfloor\frac{M}{N}\right\rfloor$ sub-blocks consists of $N$ symbols. Specifically, for the second to the $\left\lfloor\frac{M}{N}\right\rfloor^{t h}$ subblocks, the channel state remains the same in each sub-block while changes across different sub-blocks. That is, in subblock $i=2, \cdots,\left\lfloor\frac{M}{N}\right\rfloor$, the channel is $\mathbf{H}^{[1]}(i)$. In the last sub-block, the channel state changes over each symbol, i.e., $\mathbf{H}^{[1]}\left(\left\lfloor\frac{M}{N}\right\rfloor+1\right), \cdots, \mathbf{H}^{[1]}\left(\left\lfloor\frac{M}{N}\right\rfloor+N\right)$. Note that the order of symbols in the alignment block can be made arbitrarily. The SAS pattern within each $N \times M$ channel matrix in the general case is designed as follows.

$$
\begin{aligned}
\mathbf{H}^{[1]}(1) & =\left[\begin{array}{c}
\mathbf{h}_{1}^{[1]} \\
\vdots \\
\mathbf{h}_{N}^{[1]}
\end{array}\right], \quad \mathbf{H}^{[1]}(i)=\left[\begin{array}{c}
\mathbf{h}_{(i-1) N+1}^{[1]} \\
\vdots \\
\mathbf{h}_{i N}^{[1]}
\end{array}\right] i=2, \ldots,\left\lfloor\frac{M}{N}\right\rfloor \\
\mathbf{H}^{[1]}\left(\left\lfloor\frac{M}{N}\right\rfloor+i\right) & =\left[\begin{array}{c}
\mathbf{h}_{M-(M \mid N)+1}^{[1]} \\
\vdots \\
\mathbf{h}_{M]}^{[1]} \\
\mathbf{h}_{i \mid N}^{[1]} \\
\vdots \\
\mathbf{h}_{(i-1+N-(M \mid N)) \mid N}^{[1]}
\end{array}\right] i=1, \ldots, N
\end{aligned}
$$

In fact, we can draw an analogy between the alignment block of $M \times N$ MIMO BC and that of the $M^{\prime} \times 1$ MISO BC where $M^{\prime}=\left\lfloor\frac{M}{N}\right\rfloor+1$. As shown in Figure 4, if we view each sub-block as one symbol, then it is similar to the alignment block of the $M^{\prime} \times 1$ MISO BC. In other words, we can obtain the alignment block of the $M \times N$ MIMO BC from that of $M^{\prime} \times 1$ MISO BC. To do that, we need two operations, repeat and expansion. In particular, we choose the second to $\left\lfloor\frac{M}{N}\right\rfloor^{t h}$ symbols from the alignment block of the $M^{\prime} \times 1$ MISO BC and repeat each symbol $N$ times. In addition, we choose the last one symbol of the $M^{\prime} \times 1$ MISO BC and expand it to $N$ symbols with different channel values from all other symbols.

With the alignment block, we can write the beamforming matrix as a block matrix from Figure 4. This can be done in

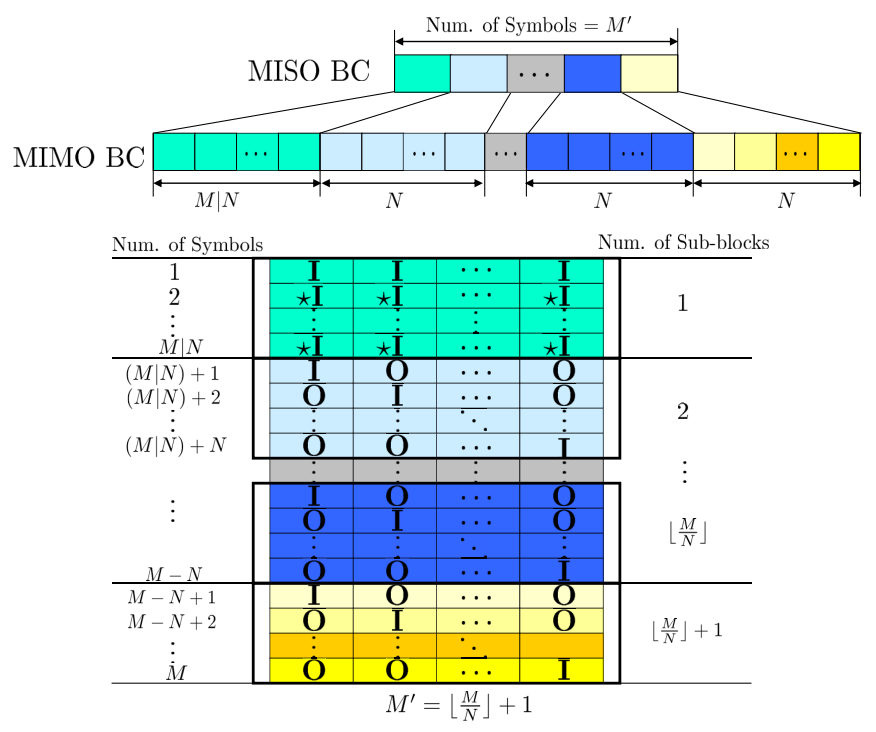

Fig. 4. Structure of one alignment block of the $M \times N$ MIMO BC 
the following form. Let us define two block $\mathbf{B}_{1}$ and $\mathbf{B}_{2}$ as

$$
\mathbf{B}_{1}=\left[\begin{array}{cccc}
\mathbf{I} & \mathbf{I} & \cdots & \mathbf{I} \\
\alpha_{21} \mathbf{I} & \alpha_{22} \mathbf{I} & \cdots & \alpha_{2 N} \mathbf{I} \\
\vdots & \vdots & \vdots & \vdots \\
\alpha_{(M \mid N) 1} \mathbf{I} & \alpha_{(M \mid N) 2} \mathbf{I} & \cdots & \alpha_{(M \mid N) N} \mathbf{I}
\end{array}\right]
$$

and

$$
\mathbf{B}_{2}=\left[\begin{array}{cccc}
\mathbf{I} & \mathbf{O} & \cdots & \mathbf{O} \\
\mathbf{O} & \mathbf{I} & \cdots & \mathbf{O} \\
\vdots & \vdots & \ddots & \vdots \\
\mathbf{O} & \mathbf{O} & \cdots & \mathbf{I}
\end{array}\right]_{M N \times M N}
$$

where $\mathbf{I}$ is the $M \times M$ identity matrix. Then, $\mathbf{B}$ can be obtained by stacking one $\mathbf{B}_{1}$ and $\left\lfloor\frac{M}{N}\right\rfloor \mathbf{B}_{2}$ :

$$
\mathbf{B}=\left[\begin{array}{c}
\mathbf{B}_{1} \\
\mathbf{B}_{2} \\
\vdots \\
\mathbf{B}_{2}
\end{array}\right]_{M^{2} \times M N}
$$

It can be easily verified that the received signal is along a full rank $M N \times M N$ matrix. We omit the rigorous proof here due to the space limitation.

With such construction, each user can achieve $M N$ DoF in one alignment block. While $M N$ interference data streams can be aligned into $N^{2}$ dimensions at all undesired users.

\section{SUPERSYMbOL MAPPING FOR $K$ USER MIMO BC}

With the alignment block, we can design the supersymbol structure by creating non-overlapping alignment blocks for each user. Similar to the MISO case, the supersymbol can be divided into two blocks. Block 1 ensures the alignment and Block 2 ensures that the desired signals do not overlap with interference [6], also shown in the example of Fig.5. Also, the key is to design Block 1. Once designed, Block 2 can be determined automatically. As mentioned before, the alignment block for the $M \times N$ MIMO BC can be obtained from that of the $M^{\prime} \times 1$ MISO BC where $M^{\prime}=\left\lfloor\frac{M}{N}\right\rfloor+1$. Since the alignment blocks constitute the supersymbol, the supersymbol of the MIMO BC can also be obtained from that of its MISO counterpart. We will first explain this through an example, then show the structure for the general case.

\section{A. 3 User $5 \times 2$ MIMO BC}

For the 3 user $5 \times 2$ MIMO BC, $M^{\prime}=3$. Thus, it corresponds to the 3 user $3 \times 1$ MISO BC. First let us consider the mapping of the alignment blocks between these two cases. Recall that the alignment block for $3 \times 1$ MISO BC consists of three symbols. From the mapping shown in Figure 5, we can obtain the alignment block for the $5 \times 2$ MIMO BC shown in Figure 2 by repeating the second symbol in the alignment block of $3 \times 1$ MISO BC twice and expand the last symbol into two symbols with different channel values. With the understanding of mapping of alignment blocks, now we consider the mapping of supersymbols from MISO case to MIMO case. In the MISO case, the supersymbol of each user consists of four alignment blocks shown in Figure 5. Thus, to

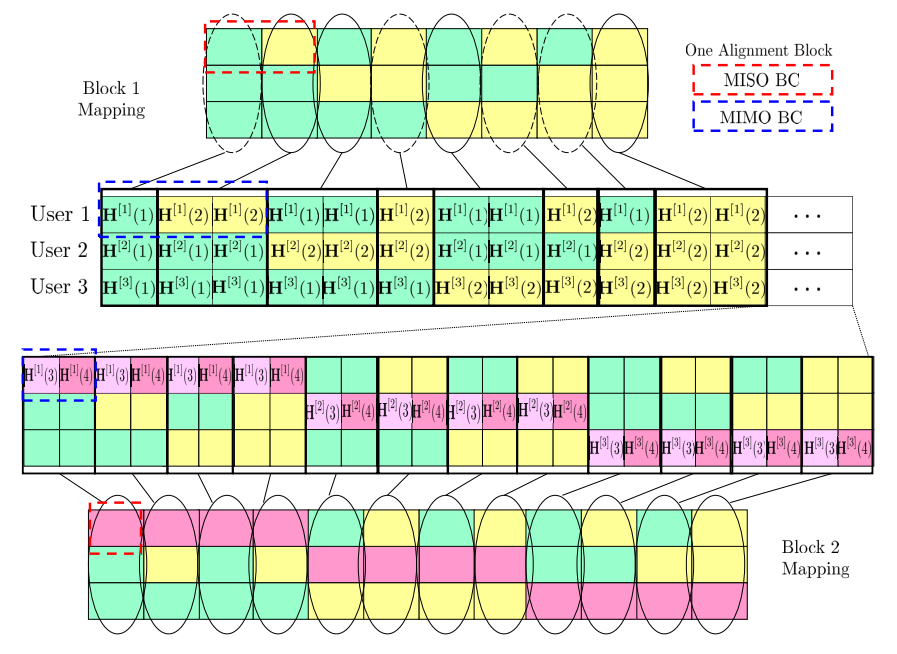

Fig. 5. Mapping of supersymbol from $3 \times 1$ MISO BC to $5 \times 2$ MIMO BC
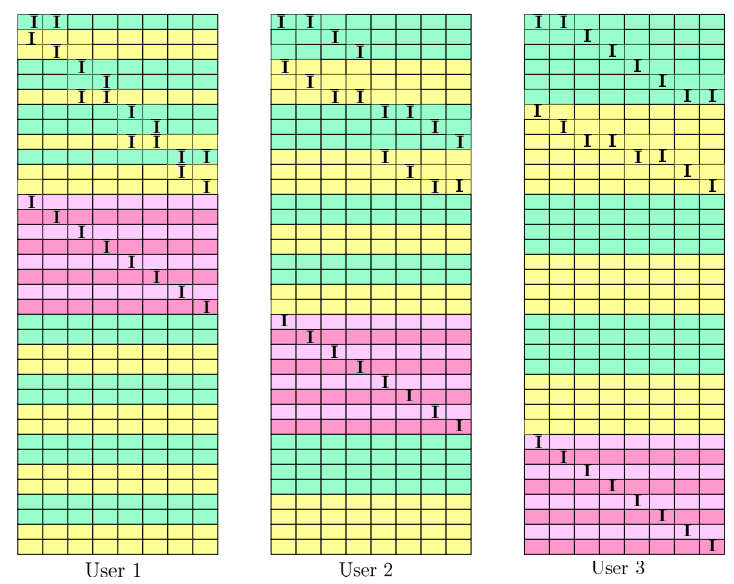

Fig. 6. The beamforming matrices for 3 user $5 \times 2$ MIMO BC

clarify the mappings, we only need to specify which symbols in each alignment block need repeating or expanding. Here we choose the last symbol of each alignment block of the MISO case to be always expanded, thus obtaining Block 2 of MIMO case. Now let us consider Block 1 of MISO case, which consists of four groups corresponding to four alignment blocks. We need to specify which one of the two symbols in each group needs repeating. Due to the interleaving structure of Block 1, it is sufficient to specify this for user 1. For user 1 's temporal signature in Block 1, there are four consecutive groups, each consisting of two different symbols. From the first to the fourth group, we choose the second, first, first and second symbol in four groups, respectively, to be expanded. Actually, the symbol chosen in $j^{t h}$ group, denoted as $c^{[j]}$, can be determined as follows. We label two symbols within each group with indices 1 and 2, respectively. We first arbitrarily choose one symbol in the first group. In this example, we choose $c^{[1]}=2$. Then the index we choose for the second group depends on $c^{[1]}$. That is, the index we choose in the 
second group can be decided through a mapping function $c^{[2]}=\left(c^{[1]} \mid\left(M^{\prime}-1\right)\right)+1=1$. After the first two groups are determined, the third and fourth groups can determine their indices based on that of the first and the second groups by the same mapping function, respectively. Specifically for $j=3,4$, we have $c^{[j]}=\left(c^{[j-2]} \mid\left(M^{\prime}-1\right)\right)+1$, i.e., $c^{[3]}=1, c^{[4]}=2$.

After designing the supersymbol, we can group alignment blocks to design the beamforming matrix. In fact, the grouping for the MIMO case is the same as its MISO counterpart. The beamforming matrices for the 3 user $5 \times 2$ MIMO $\mathrm{BC}$ are shown in Figure 6.

\section{B. $K$ User $M \times N$ MIMO BC}

With the understanding of the example above, now we show how to construct the supersymbol for the $K$ user $M \times N$ MIMO BC from that of the $M^{\prime} \times 1$ MISO BC where $M^{\prime}=\left\lfloor\frac{M}{N}\right\rfloor+1$. First let us consider the mapping between the alignment blocks as shown in Figure 4. As mentioned before, for each alignment block, the last symbol is always chosen to expand for $N$ symbols with different channel values. Besides this, we still need to choose $\left\lfloor\frac{M}{N}\right\rfloor-1$ out of the remaining $M^{\prime}-1$ symbols to repeat $N$ times for each symbol. Since the last symbol constitutes Block 2 of the supersymbol, we can easily obtain it for the $K$ user $M \times N$ MIMO BC after expanding the last symbol of every alignment block for every user in the supersymbol of the $K$ user $M^{\prime} \times 1$ MISO BC. Thus, the remaining issue is to design Block 1. In Block 1 of supersymbol of $K$ user $M \times 1$ MISO BC, there are $\left(M^{\prime}-1\right)^{K-1}$ groups for each user, and we need to specify which $\left\lfloor\frac{M}{N}\right\rfloor-1$ symbols we choose for repeating in each group. Due to the interleaving structure of Block 1, it is sufficient to specify this for user 1 .

For user 1, in each of $\left(M^{\prime}-1\right)^{K-1}$ groups, we label $M^{\prime}-1$ symbols with indices 1 to $M^{\prime}-1$, respectively. Then we need to choose $\left\lfloor\frac{M}{N}\right\rfloor-1=M^{\prime}-2$ indices for each group. Note that if $\left\lfloor\frac{M}{N}\right\rfloor-1=0$, then there is no need to repeat. Thus, in this case, Block 1 is the same for MISO and MIMO cases. Now we consider $\left\lfloor\frac{M}{N}\right\rfloor-1 \neq 0$. Let $c_{i}^{[j]} \in\left\{1, \ldots, M^{\prime}-1\right\}$ denote the index of the $i^{t h}, i=1, \ldots, M^{\prime}-2$, symbol chosen in the $j^{t h}$ group, $j=1, \ldots,\left(M^{\prime}-1\right)^{K-1}$. The indices which are chosen in each group are as follows.

In the first group, the indices we choose are $2,3, \ldots, M^{\prime}-1$, i.e, $c_{i}^{[1]}=i+1$. Once the first group is specified, we can determine the second to the $\left(M^{\prime}-1\right)^{t h}$ groups. The index in each group is obtained by cyclic shifting one digit to the right of its previous group. Mathematically, we have $c_{i}^{[n]}=\left(c_{i}^{[n-1]}\right.$ $\left.\bmod \left(M^{\prime}-1\right)\right)+1, n=2, \ldots M^{\prime}-1$. Based on the first $M^{\prime}-1$ groups, we can specify the first $\left(M^{\prime}-1\right)^{2}$ groups which can be divided into $M^{\prime}-1$ blocks, each of which has $M^{\prime}-1$ groups. Again, indices of each group in each block is one digit cyclic shift of those of its corresponding group in the previous block. Mathematically, we have the iterative mapping function

$$
\begin{aligned}
c_{i}^{\left[n+k\left(M^{\prime}-1\right)\right]} & =\left(c_{i}^{\left[n+(k-1)\left(M^{\prime}-1\right)\right]} \mid\left(M^{\prime}-1\right)\right)+1 \\
k=1, \ldots, M^{\prime}-1, \quad n & =1, \ldots, M^{\prime}-1, \quad i=1, \ldots, M^{\prime}-2 .
\end{aligned}
$$

In general, in order to decide the indices of the first $\left(M^{\prime}-\right.$ $1)^{l}, l=1, \ldots, K$ blocks, we need to know the first $\left(M^{\prime}-1\right)^{l-1}$ blocks. The relation is specified by the following function.

$$
\begin{aligned}
c_{i}^{\left[n+k\left(M^{\prime}-1\right)^{l-1}\right]}=\left(c_{i}^{\left[n+(k-1)\left(M^{\prime}-1\right)^{l-1}\right]} \mid\left(M^{\prime}-1\right)\right)+1 \\
k=1, \ldots, M^{\prime}-1, \quad n=1, \ldots,\left(M^{\prime}-1\right)^{l-1}, \quad i=1, \ldots, M^{\prime}-2 .
\end{aligned}
$$

After obtaining the supersymbol, we can group symbols to create alignment blocks for each user. The grouping for the MIMO case is the same as its MISO counterpart. After alignment blocks are designed, we can determine the beamforming matrix corresponding to each alignment block according to the mapping shown in Figure 4.

Now we can calculate the DoF achieved using this scheme. For each user, over one alignment block, $M N$ DoF can be achieved for the desired user while interference is aligned into $N^{2}$ dimensions. For $K$ user $M \times N$ MIMO BC, each user has $\left(M^{\prime}-1\right)^{K-1}$ alignment blocks. So for each user, the desired signal occupies $M N\left(M^{\prime}-1\right)^{K-1}$ dimensions. At each receiver, the interference occupies $N^{2}\left(M^{\prime}-1\right)^{K-1}(K-1)$ dimensions. Now consider the number of symbol extensions. In Block 1 of the supersymbol, there are $\left(M^{\prime}-1\right)^{K-1}(M-N)$ symbols. In Block 2, there are $K\left(M^{\prime}-1\right)^{K-1} N$ symbols. Therefore, the total number of symbol extensions is $\left(M^{\prime}-\right.$ $1)^{K-1}(K N+M-N)$. Since each receiver has $N$ antennas, the signal space at each receiver has $N\left(M^{\prime}-1\right)^{K-1}(K N+M-$ $N)$ dimensions which is equal to the total dimensions occupied by the desired signal and interference. Since interference and the desired signal do not overlap with each other, each user can achieve $M N\left(M^{\prime}-1\right)^{K-1}$ DoF. Thus, the normalized total number of DoF is $\frac{M N\left(M^{\prime}-1\right)^{K-1} K}{\left(M^{\prime}-1\right)^{K-1}(K N+M-N)}=\frac{M N K}{K N+M-N}$.

\section{CONClusion}

We extend the DoF result for MISO BC in [6] to MIMO BC. In this paper, we propose a systematic way to achieve blind interference alignment for the $K$ user $M \times N$ MIMO $\mathrm{BC}$. In the procedure of designing the supersymbol structure of MIMO BC, there are two main issues to handle - SAS patterns and the supersymbol mapping from MISO BC to MIMO BC. We demonstrate these two points in details in this paper. Again, the proposed scheme does not require the knowledge of channel values at the transmitter to align interference nor at receivers to cancel interference. Thus, our scheme is a promising candidate to increase the achievable rate of the wireless network in practice.

\section{REFERENCES}

[1] H. Weingarten, S. Shamai and G. Kramer, "On the compound MIMO broadcast channel", Information Theory Workshop, Jan. 2007

[2] T. Gou, S. Jafar and C. Wang, "On the Degrees of Freedom of Finite State Compound Wireless Networks - Settling a Conjecture by Weingarten et. al", arXiv:0909.4177, 2009.

[3] C. Huang, S. Jafar, S. Shamai, S. Vishwanath, "On Degrees of Freedom Region of MIMO Networks without CSIT", e-print arXiv:0909.4017, Sept. 2009.

[4] S. Jafar, "Exploiting Channel Correlations - Simple Interference Alignment Schemes with no CSIT", arXiv:0910.0555, 2009.

[5] Mohammad Ali Maddah-Ali, "On the Degrees of Freedom of the Compound MIMO Broadcast Channels with Finite States", arXiv:0909.5006, 2009

[6] C. Wang, T. Gou and S. Jafar, "Aiming Perfectly in the Dark Blind Interference Alignment through Staggered Antenna Switching?", http://arxiv.org/abs/1002.2720, Feb. 2010. 